\title{
Relación investigación, innovación: el desarrollo de las empresas ecuatorianas con las universidades
}

\section{Research, innovation: the development of Ecuadorian companies with universities}

Luis Barreno Benavides ${ }^{1}$, Héctor López Paredes ${ }^{2}$, Marco López Paredes ${ }^{3}$

INFORMACIÓN DEL ARTÍCULO

Fecha de recepción: 5 de Marzo de 2018

Fecha de aceptación: 26 de Abril de 2018

\footnotetext{
1 MBA, Universidad De Matanzas Camilo Cienfuegos. Docente e Investigador de la Universidad Tecnológica Equinoccial-Ecuador. E-mail: lbarreno@ute.edu.ec
}

$2 \mathrm{PhDc}$ en Dirección de Empresas, Universidad de Valencia. Docente e Investigador de la Universidad Tecnológica Equinoccial-Ecuador. E-mail: hector.lopez@ute.edu.ec

${ }_{3}^{3} \mathrm{PhD}$ en Comunicación, Universidad de Artes Ciencias y Comunicación. Docente Principal Investigador de la Pontificia Universidad Católica -Ecuador.

E-mail: mvlopez@puce.edu.ec

CITACIÓN: Barreno Benavides, L., López Paredes, H., \& López Paredes, M. (2018). Relación investigación, innovación: el desarrollo de las empresas ecuatorianas con las universidades. Podium, 33, 55-68 doi:10.31095/podium.2018.33.6

ENLACE DOI

http://dx.doi.org/10.31095/podium.201 8.33 .6

\section{Resumen}

Este artículo tiene por objeto analizar la relación existente en la investigación, la innovación y el desarrollo de las empresas ecuatorianas con las Instituciones de Educación Superior (IES). La investigación fue exploratoria, descriptiva y analítica, aplicando una encuesta a 385 empresas públicas y privadas de las provincias de Pichincha, Santo Domingo de los Tsáchilas y Santa Elena, para revisar experiencias de vinculación con IES en la ejecución de proyectos de investigación; así como, la inversión realizada por países de la región y sus fuentes de financiamiento. Los resultados muestran la necesidad de transferir conocimiento para el desarrollo empresarial y social, evidenciando sectores que demandan de investigación para la innovación, que la inversión para investigación e innovación es un elemento fundamental para estimular la producción, la generación de empleo y la creación de nuevos emprendimientos, y se destaca la importancia de vincular la universidad con la empresa para generar desarrollo.

\section{Palabras Clave:}

Investigación, Innovación, Desarrollo económico y social, Empresa, Educación.

Clasificación JEL: H75, I23, O31.

Abstract

The purpose of this paper is to analyze the relationship that exists between higher education institutions (HEI) and the research, innovation and development of Ecuadorian companies. Research was exploratory, descriptive and analytic; a survey of 385 public and private companies in the provinces of Pichincha, Santo Domingo de los Tsáchilas and Santa Elena was used in order to review the experience of linking companies with higher education institutions for the execution of research projects; the investment made by the region's countries and their sources of financing was also reviewed. Results show (1) the need for knowledge transfer for the development of business and society, evidencing sectors that demand innovation research; (2) it shows that research investment is a fundamental element to stimulate production, to generate employment generation and to create new ventures. The importance of establishing a link between universities and business in order to generate development is insisted on (3).

Keywords:

Research, Innovation, Development, Company, Education.

JEL Classification: H75, I23, $\mathrm{O} 31$. 


\section{Introducción}

Una de las variables más importantes que permite el crecimiento y desarrollo económico y social de los países es la investigación permanente en procesos innovadores para los sectores de la producción, comercialización y servicios. Si bien son las empresas privadas, en primer término, las encargadas de realizar $\mathrm{y}$ aplicar los resultados de las investigaciones, no es menos cierto que es obligación de los centros de educación superior contribuir con estas, de ahí que los vínculos entre estas organizaciones deban ser directos y permanentes en la búsqueda de alternativas que permitan producir bienes y servicios con altos niveles de calidad al menor costo posible, lo que beneficiará a toda la sociedad.

Con lo expuesto, la presente investigación tuvo por objeto analizar la relación existente en la investigación, la innovación y el desarrollo de las empresas ecuatorianas con las Instituciones de Educación Superior (IES). Para ello se recabó información de campo, apoyada con investigación documental, y se establecieron relaciones y conclusiones a partir de la información analizada.

\section{Investigación en IES}

El entender el rol de la investigación en las IES debe suponer qué la origina y en qué favorece el realizarla, pues según Colina (2007) la pertinencia social de la oferta académica de una universidad se debe abordar desde una perspectiva que trascienda el carácter utilitarista, centrándose en aportar a las demandas de la economía o del sector productivo, pues la investigación constituye un eje fundamental para la generación de conocimiento en respuesta a las interrogantes expuestas por el hombre para mejorar su condición de vida. Carvajal (2015) manifiesta que la investigación es un proceso natural, intelectual $\mathrm{y}$ experimental, que a través de un proceso sistémico y metódico persigue indagar sobre un asunto o tema, coincidiendo con lo expresado por Rojas (2002), en cuanto a que la investigación científica no es otra cosa que la actividad intelectual y práctica debidamente estructurada que se apoya en teorías e hipótesis y utiliza técnicas e instrumentos de recolección y análisis de los datos, con el objeto de conocer objetivamente las causas y consecuencias o la modalidad que adoptan los fenómenos en determinado momento o durante el transcurso del tiempo.

Morone (s.f.) señala que, la investigación científica es la encargada de producir conocimiento que se caracteriza por ser ordenado, sistémico, metódico, racional / reflexivo, crítico / subversivo en respuesta a una problemática planteada; por lo cual, se puede concluir que la investigación científica permite la construcción de nuevos conocimientos para obtener nuevos productos, procesos o mejorar los existentes en todos los ámbitos de formación, razón por la cual los resultados de esta investigación permiten, por ejemplo, comprender la situación de las IES en cuanto a su producción investigativa, su relación con el desarrollo empresarial y su impulso con la innovación, así como contar con información que apalanque el proceso 
administrativo y dentro de este el de control en las instituciones de educación superior.

En relación a la investigación generada en las IES de Ecuador se hace la siguiente referencia:

...hasta los años setenta la universidades en el pais se enfocaban únicamente a la docencia dejando poco espacio a la investigación, existiendo pocas publicaciones que representaban la recopilación de discursos y ensayos, al igual que se generaban tesis en su mayoría con temáticas repetitivas, dejando el saber científico a los aportes extranjeros, con lo cual a partir de esta decana se generaron varios cambios legales y estructurales con el afán de incentivar la producción científica en beneficio de la sociedad, muestra de ello son los cambios generados por las instituciones de educación superior en adelante (IES) quienes se han visto en la obligación de contratar profesionales a tiempo completo y medio tiempo con requisitos de formación elevados y con capacidad de generar propuestas de investigación de alto nivel que lleven a las IES a cumplir con requisitos de acreditación y posicionamiento, situación que en los últimos años llevó al cierre de varias instituciones y la intervención de otras tantas. (Ayala, 2015, p.2)

Se debe pensar en lo manifestado por Colina (2007) respecto a la necesidad de enfocar la investigación académica a los criterios de búsqueda de resultados socialmente pertinentes, orientados por una estrategia de investigación que conduzca a la obtención de contenidos válidos o relevantes para la sociedad, en donde, considerando lo expresado por Dallanegra (2004) luego de la caída del Muro de Berlín en 1989 y la desintegración de la URSS en 1991, el mundo ingresó de lleno en la era del conocimiento, siendo la materia prima más preciada la "materia gris", pues en los países más desarrollados la educación y la investigación científica constituyen áreas estratégicas y les destinan porcentajes importantes de sus PIB.

\section{La innovación en las empresas}

Garzón Castrillón e Ibarra Mares (2013) hacen referencia a que la innovación ha sido descrita y definida en diferentes formas y sentidos.

La innovación responde a la aplicación de un método de producción o servicio nuevo o significativamente mejorado que incluyan cambios representativos en cuanto a técnicas, equipos, programas informáticos incluyendo innovación tecnológica en materia de procesos, aplicación de nuevos métodos organizacionales a las prácticas comerciales y a la organización en el centro de trabajo o las relaciones exteriores de la empresa, etc; se lo denomina innovación. (Cámara Zaragoza, 2011).

González Molano y Martínez Campo (2014) señalan la necesidad que tienen las empresas de contar con metodologías y herramientas para evaluar su capacidad de gestión de la innovación, con miras a intervenir oportuna $y$ asertivamente mediante la gestión de proyectos de innovación que respondan con sus propósitos estratégicos, en procura de 
hacer las empresas más competitivas en sus respectivos mercados (Ver figura 1).

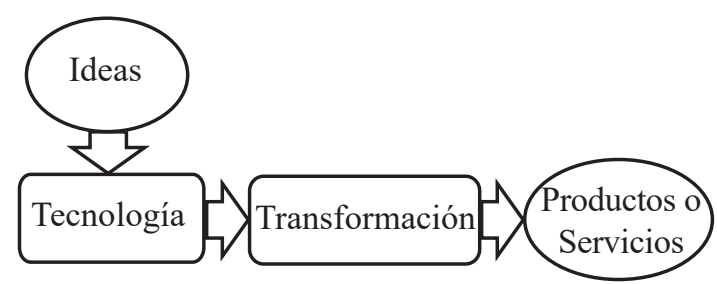

Figura 1. Proceso básico de innovación

Fuente: González Molano y Martínez Campo (2014)

Berumen (2011) manifiesta que la empresa es el agente innovador insustituible, transformador dentro de un entorno con el que interactúa convirtiéndose en facilitador $\mathrm{u}$ obstaculizador de la introducción de nuevos productos, procesos o cambios organizacionales. De hecho, el autor considera que la empresa es el ambiente propicio para realizar las investigaciones que permitan obtener resultados renovadores para la creación de nuevos productos o servicios o a su vez mejorar los existentes, señalando que la innovación debe darse en todos los procesos: productivos, comerciales $\mathrm{y}$ administrativo financieros.

Muchas han sido las aplicaciones que las empresas han realizado de los productos de investigación para innovación en todo el mundo (Garzón Castrillón e Ibarra Mares, 2013; González Molano y Martínez Campo, 2014; CEPAL, 2010) bien en procesos de producción, comercialización, operación, otros un tanto más especializados como la producción de hormonas para uso animal, la producción de factores de crecimiento vegetal o de enzimas para la agroindustria,

${ }^{4}$ Es el principal componente de la pared celular de las plantas, esta biomasa producida por la fotosíntesis es la fuente de carbono renovable más prometedora para solucionar los problemas actuales de energía. residuos lingnocelulósticos ${ }^{4}$ y forestales, cultivo masivo de microalgas, manipulación de embriones animales, anticuerpos monoclonales, biología molecular, bioseguridad alimentaria, en genética vegetal y animal, parques tecnológicos, patentes entre otros.

Garzón Castrillón e Ibarra Mares (2013) en base a su estudio realizado en relación a la innovación empresarial refiere varios tipos, uno de ellos es el del Manual de Osorio 2006, en el cual su tipología hace referencia al proceso, la función, el tiempo, el sistema social, y la creación o introducción, lo que permite entender el alcance de la innovación dentro de una empresa, es decir, justificar su impacto en el todo y no sólo en las partes.

\section{Metodología}

En función del propósito del artículo, la investigación fue de tipo exploratoria descriptiva y analítica. El desarrollo del marco teórico se basó en información secundaria validada por los autores sobre la relación entre la investigación, la innovación, el crecimiento y desarrollo en las empresas productoras de bienes materiales y servicios. La información estadística permitió complementar el análisis sobre las inversiones que el Ecuador ha realizado en investigación aplicada y científica, con financiamiento del SENESCYT y dentro de esta aquella que se ha realizado por los centros de educación superior como por distintas entidades del sector público y privado.

Para la recopilación de datos se aplicó una encuesta longitudinal, que empleó un 
cuestionario, de consulta sobre las actividades de investigación realizadas por las empresas para mejorar su crecimiento y desarrollo, y si estas fueron realizadas en convenio o con ayuda de los centros de educación superior. El mercado objetivo estuvo comprendido por un aproximado de 60.000 empresas registradas en la Superintendencia de Compañías del Ecuador, dentro del ámbito geográfico donde la Universidad Tecnológica Equinoccial (UTE) tiene sus sedes, correspondiendo a tres provincias del territorio nacional: Pichincha, Santo Domingo de los Tsáchilas y Santa Elena, y dentro de estas en su orden en las ciudades de Quito, Santo Domingo, La Libertad, Santa Elena y Salinas. Para la investigación se aplicó un muestreo aleatorio simple estratificado proporcionalmente en función del número de empresas por provincia, con un nivel de confianza del $95 \%$ y un error del $5 \%$, obteniendo una muestra de 385 empresas.

\section{Resultados}

La experiencia ecuatoriana en investigación está centrada en gran parte en las IES públicas y en los centros de educación superior financiados por el SENESCYT, respaldando la bibliografía revisada respecto al rol fundamental del Estado para impulsar el desarrollo social a través de la educación, explorando la situación del Ecuador, para después compararla con la realidad de la región. En la Figura 2 se muestra la inversión en investigación en el Ecuador, financiada con recursos del presupuesto del Estado a través del SENESCYT donde un 59\% corresponde a los institutos públicos. El mayor porcentaje de las investigaciones realizadas por los centros de educación superior lo realizan los de tipo público.

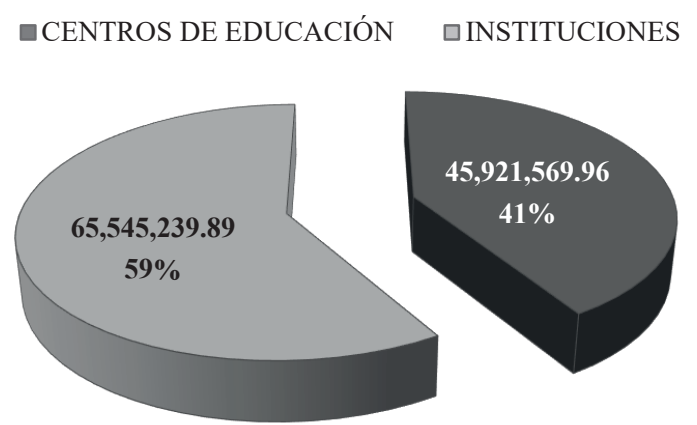

Figura 2. Inversiones en investigación financiadas por la SENESCYT. Adaptado de la base de datos facilitada por la SENESCYT del período enero a diciembre 2016.

Como se muestra en la Figura 3, de las investigaciones realizadas por los centros de educación superior, el $87 \%$ lo realizan los públicos y apenas un 13\% los privados, siendo fundamental reflexionar sobre lo planteado por Dallanegra (2004) respecto al papel fundamental que debe desempeñar la educación y con ello la investigación para el desarrollo de un país, a lo cual se suma la necesidad de control del Estado para equilibrar el esfuerzo de las IES públicas y privadas. El $96.3 \%$ de la inversión en investigación se concentra en siete centros y el $3.7 \%$ en el grupo otras, que agrupa siete IES, las demás no tienen ninguna participación, aspecto que debe tratarse desde los planteado por Ayala (2015) al igual que lo mencionado por CEPAL (2010) en cuanto a la responsabilidad de las IES de impulsar cambios significativos en los modelos de desarrollo social, así como, la importancia de transferir los resultados de sus investigaciones. Sin embargo, la realidad del Ecuador muestra gran diferencia entre las IES públicas respecto de las privadas, lo 


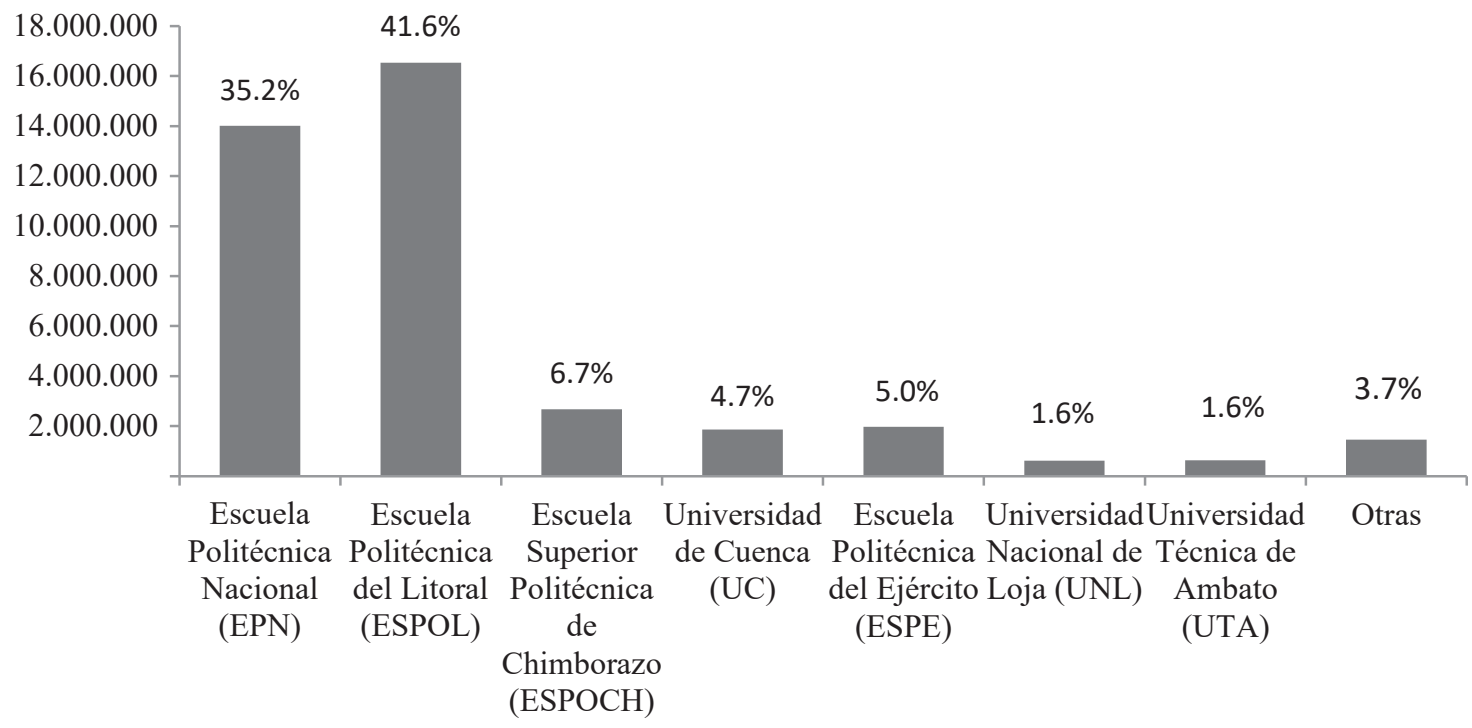

Figura 3. Inversiones en dólares de IES públicas en investigación. Tomado de la base de datos facilitada por la SENESCYT del período enero a diciembre 2016.

que debe ser tratado desde los entes de control para responder y articular las necesidades presentes.

En el grupo de IES privadas o particulares que invierten en investigación, el $85 \%$ se concentra en 3 de ellas (Ver figura 4). A esta situación se incorpora la realidad país manifestada por Ayala (2015) relacionada también con lo expuesto por Colina (2007) referente al espacio en el que se genera la oferta académica por parte de las IES con un respaldo de negocio sin base social, situación que en la realidad ecuatoriana al igual que en la latinoamericana, ha incentivado la creación de universidades privadas con poca infraestructura que han centrado su servicio en docencia, sin considerar el elemento fundamental de la

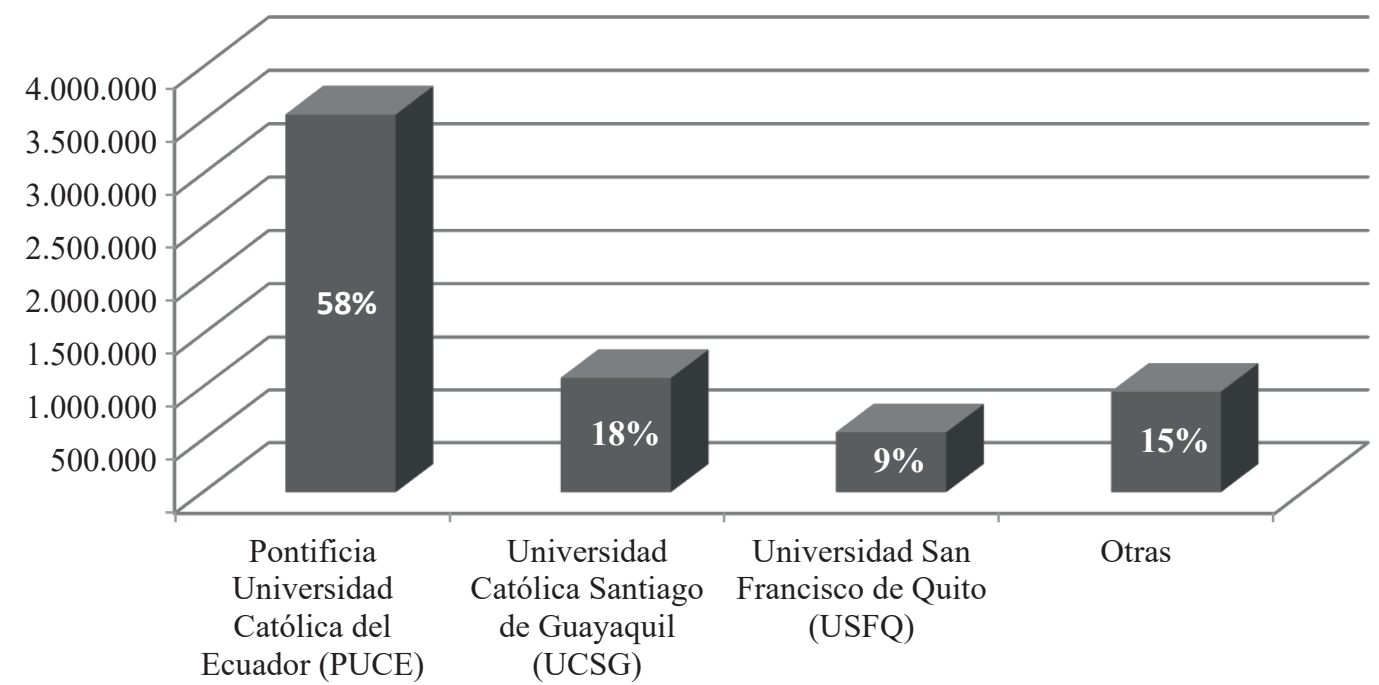

Figura 4. Inversiones en dólares de IES privada en investigación. Tomado de la base de datos facilitada por la SENESCYT del período enero a diciembre 2016. 


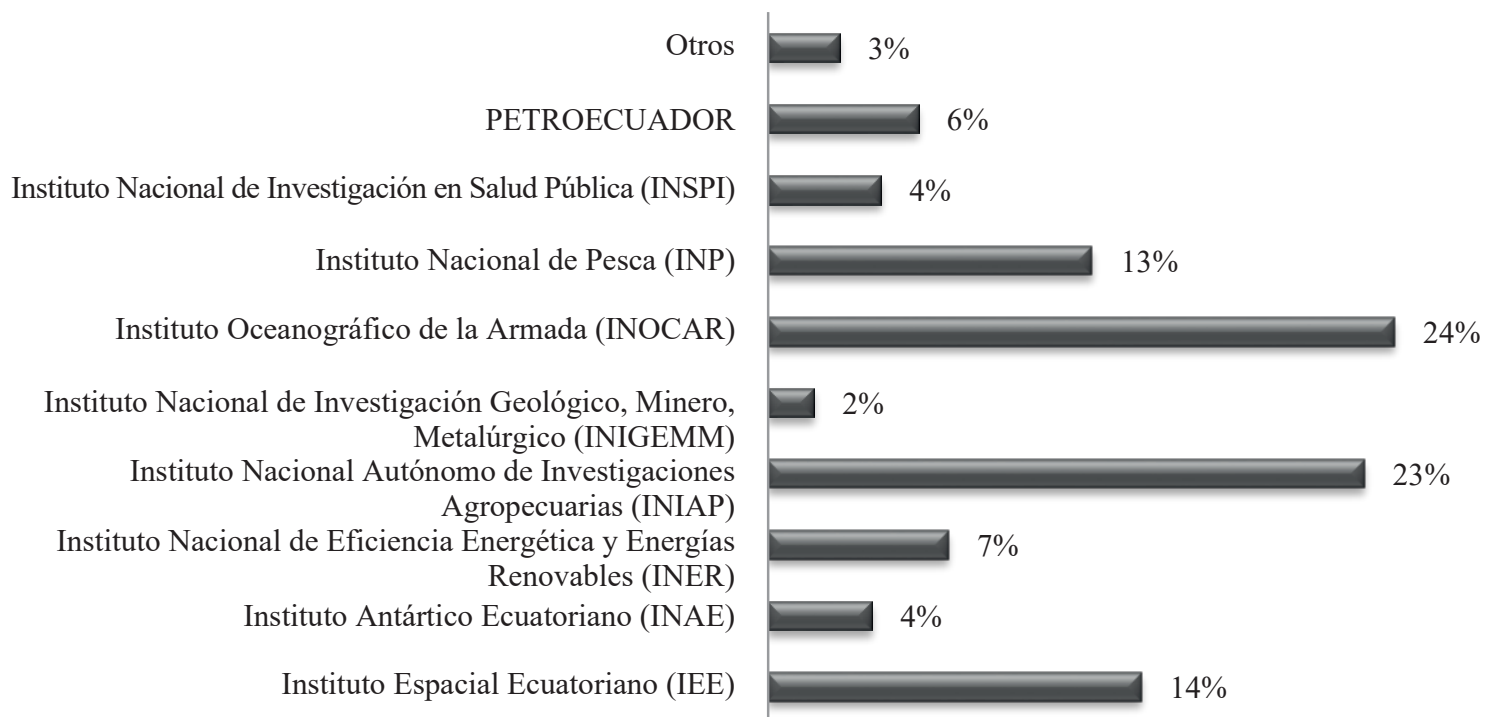

Figura 5. Inversiones de Institutos públicos. Adaptado de la base de datos facilitada por la SENESCYT del período enero a diciembre 2016.

educación como es la investigación.

En relación con las investigaciones realizadas por las IES, el $99.7 \%$ son realizadas por las públicas y apenas el $0.3 \%$ por las privadas. Esto evidencia la falta de producción científica de las IES ecuatorianas en respuesta de las necesidades latentes de desarrollo, producción, empleo y demás factores analizados permanentemente por CEPAL (2010) y discutidos por Colina (2007) y Cárdenas, Cabrero, y Arellano (2014).

En el caso de los Institutos se mantiene la realidad ya expresada en las IES públicas, reflejando la persistencia de los problemas relacionados a investigación desde la formación técnica (Ver figura 5).

\section{Situación actual de la investigación e innovación en las empresas ecuatorianas}

Esta sección corresponde a los resultados obtenidos del estudio sobre los requerimientos de Investigación y Vinculación en empresas privadas y públicas, considerando que las empresas investigadas son de diferentes sectores, esto es, producción, servicios, comercio, o la combinación de estas, guardando una participación similar estructura empresarial por tipo de actividad del Ecuador (Ver tabla 1).

Tabla 1.

Distribución de las empresas analizadas por actividad

\begin{tabular}{lcc}
\hline $\begin{array}{c}\text { Actividad de la } \\
\text { empresa }\end{array}$ & $\begin{array}{c}\text { Número de } \\
\text { empresas }\end{array}$ & Porcentaje \\
\hline Producción & 57 & 14.9 \\
Servicios & 212 & 55.1 \\
Comercialización & 116 & 30.0 \\
Total & 385 & 100.0 \\
\hline
\end{tabular}

Fuente: Elaborado por los autores.

Un 7\% corresponde a empresas que ofrecen servicios a la comunidad, siendo esto a las empresas y entidades del sector público, $5 \%$ el servicio de salud, $1 \%$ las que venden calzado y ropa, 
telecomunicaciones con el $0.8 \%$; y a continuación existe una gran cantidad de empresas que ofertan diversos productos casi en todas las líneas de productos existentes. Para determinar el tamaño de la empresa se consideró el número de empleados y trabajadores, incluyéndose en el estudio empresas privadas que tienen desde 4 hasta 9000 trabajadores y organizaciones públicas con hasta 7719 trabajadores.

Apenas el 17\% de las empresas han realizado alguna actividad de Investigación, Desarrollo Tecnológico o Innovación, confirmándose lo afirmado inicialmente sobre la escasa actividad investigativa que existe en las empresas ecuatorianas, tanto del sector público como privadas, haciendo imprescindible la necesidad de construir vínculos de los centros de educación superior con las organizaciones productivas privadas y las públicas que emiten políticas de coordinación y control respecto a la articulación de los factores de evolución y desarrollo de los países (Garzón Castrillón y Ibarra Mares, 2013; Ayala, 2015; Colina, 2007; Cárdenas et al, 2014).

\section{Vínculo entre las empresas y los centros de educación superior}

La experiencia internacional muestra que los países donde las empresas y los centros de investigación e instituciones de educación superior han podido vincularse intensa y positivamente, han avanzado más rápido en la economía del conocimiento, $\mathrm{y}$ son los líderes en el ámbito internacional con empresas que son capaces de innovar, de desarrollar nuevos procesos tecnológicos y nuevos productos. De esta forma las empresas tienen la posibilidad innovar a partir de la adopción y la adaptación de nuevos conocimientos, procesos, patentes y sistemas de producción gestados en el sector científico y académico; y por su lado las instituciones educativas y centros científicos han podido concentrar buena parte de sus habilidades en la resolución y avances de los procesos y tecnologías de producción de las empresas (Cárdenas et al, 2014). En el marco de las interrelaciones que componen un Sistema Nacional de Investigación, las IES y las empresas son actores clave por su capacidad de trasmitir y crear conocimiento, así como de movilizar recursos para enfrentar problemas crecientemente complejos (CEPAL, 2010).

\section{Financiamiento de los proyectos de investigación y desarrollo en algunos países en el mundo}

En contraste con datos de la CEPAL (2015) en la mayoría de los países analizados de la región, los porcentajes de financiamiento para los proyectos de investigación y vinculación, se encuentran en el $50 \%$, mostrando que en la mayoría de los países el aporte de los centros de educación superior es muy limitado, a excepción de el Salvador, Guatemala, Colombia y Bolivia, en donde el porcentaje de financiamiento bordea el 30\%. Los países que lideran el aporte a las empresas de carácter privado son: la República de Corea, Estados Unidos, Finlandia, y México, alcanzando porcentajes desde el 60 hasta el 70\%. 
El aporte del financiamiento internacional a través de cooperación técnica es importante especialmente en los países centroamericanos donde este es significativo como en los casos de Panamá y Guatemala, manteniendo relación entre la investigación, el desarrollo empresarial y el desarrollo país, entendiendo que a mayor inversión en investigación, mayor desarrollo de las empresas, y a mayor desarrollo de las empresas, mayor desarrollo país (Ver tabla 2).

Tabla 2.

Distribución del financiamiento internacional para la investigación y desarrollo por países.

\begin{tabular}{|c|c|c|c|c|c|c|c|c|c|}
\hline Ecuador & & & & & & & & & \\
\hline Costa Rica & & & & & & & & & \\
\hline Paraguay & & & & & & & & & \\
\hline Cuba & & & & & & & & & \\
\hline Argentina & & & & & & & & & \\
\hline Uruguay & & & & & & & & & \\
\hline Brasil & & & & & & & & & \\
\hline México & & & & & & & & & \\
\hline El Salvado & & & & & & & & & \\
\hline España & & & & & & & & & \\
\hline Panamá & & & & & & & & & \\
\hline Chile & & & & & & & & & \\
\hline Portugal & & & & & & & & & \\
\hline Colombia & & & & & & & & & \\
\hline Guatemala & & & & & & & & & \\
\hline Bolivia & & & & & & & & & \\
\hline $\begin{array}{l}\text { Estados } \\
\text { Unidos }\end{array}$ & & & & & & & & & \\
\hline Finlandia & & & & & & & & & \\
\hline $\begin{array}{l}\text { República } \\
\text { de Corea }\end{array}$ & & & & & & & & & \\
\hline & \begin{tabular}{l|l}
0 & 10 \\
\end{tabular} & 20 & 30 & 40 & 50 & 60 & 70 & 80 & 90 \\
\hline
\end{tabular}

Fuente: Elaborado por los autores en base a la Información obtenida de la CEPAL a diciembre del 2015.

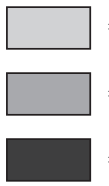

$=$ Presupuesto del Estado

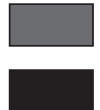

$=$ Recursos propios empresas

$=$ Recursos de instituciones de educación superior

$=$ recursos de ONGs

$=$ Financiamiento externo

\section{3}

PODIUM No. 33, Junio 2018, pp. 55-68

Universidad Espíritu Santo - UEES

ISSN: 1390-5473 e-ISSN: 2588-0969 


\section{Temas de investigación requeridos por las empresas ecuatorianas}

En la Tabla 3 se presentan las principales necesidades de investigación de las empresas participantes del estudio.

Tabla 3 .

Necesidades de investigación de las empresas investigadas distribuidas por sector

\begin{tabular}{|c|c|c|c|}
\hline SECTOR & Temas de interés & SECTOR & Temas de interés \\
\hline \multirow{10}{*}{ Alimentos } & $\begin{array}{l}\text { Manipulación posterior } \\
\text { del añejamiento }\end{array}$ & \multirow{6}{*}{$\begin{array}{c}\text { Sector } \\
\text { Automotriz }\end{array}$} & $\begin{array}{l}\text { Sistema de control de } \\
\text { emisión de gases }\end{array}$ \\
\hline & $\begin{array}{l}\text { Conservación de } \\
\text { materia prima }\end{array}$ & & Mecatrónica \\
\hline & $\begin{array}{l}\text { Trazabilidad en la } \\
\text { industria de carnes }\end{array}$ & & $\begin{array}{c}\text { Desarrollo de } \\
\text { combustibles alternativos }\end{array}$ \\
\hline & $\begin{array}{l}\text { Conservación } \\
\text { de alimentos }\end{array}$ & & Bio combustible \\
\hline & $\begin{array}{l}\text { Sabores ingredientes } \\
\text { alimenticios }\end{array}$ & & $\begin{array}{l}\text { Uso de subproductos agrícolas } \\
\text { o desechos orgánicos }\end{array}$ \\
\hline & $\begin{array}{l}\text { Nuevas variedades de } \\
\text { cacao no investigadas }\end{array}$ & & Combustibles alternativos \\
\hline & $\begin{array}{l}\text { Seguridad alimentaria } \\
\text { / desarrollo de productos }\end{array}$ & \multirow{11}{*}{$\begin{array}{c}\text { Sector } \\
\text { Ambiental }\end{array}$} & $\begin{array}{l}\text { Diagnostico o línea base de la } \\
\text { contaminación por los buses }\end{array}$ \\
\hline & $\begin{array}{c}\text { Proteínas o } \\
\text { suplementos alimenticios }\end{array}$ & & $\begin{array}{c}\text { Reparación de } \\
\text { suelos contaminados }\end{array}$ \\
\hline & $\begin{array}{c}\text { Tecnología y } \\
\text { calidad de la carne } \\
\end{array}$ & & $\begin{array}{l}\text { Gestión de desechos } \\
\text { industriales }\end{array}$ \\
\hline & $\begin{array}{c}\text { Investigación y desarrollo } \\
\text { de nuevos productos }\end{array}$ & & $\begin{array}{c}\text { Producción } \\
\text { cero residuos }\end{array}$ \\
\hline \multirow{9}{*}{ Agropecuario } & Materiales genéticos & & $\begin{array}{l}\text { Reprocesamiento } \\
\text { de residuos }\end{array}$ \\
\hline & $\begin{array}{l}\text { Reemplazo } \\
\text { de químicos }\end{array}$ & & $\begin{array}{c}\text { Estudio de impacto } \\
\text { ambiental en la construcción }\end{array}$ \\
\hline & $\begin{array}{l}\text { Nuevos componentes } \\
\text { para productos }\end{array}$ & & Producción cero residuos \\
\hline & $\begin{array}{l}\text { Aprovechamiento de la productividad } \\
\text { y optimización de recursos }\end{array}$ & & $\begin{array}{c}\text { Reprocesamiento } \\
\text { de residuos }\end{array}$ \\
\hline & Manejo orgánico & & $\begin{array}{l}\text { Estudio de identificación de } \\
\text { asentamientos de comunas } \\
\text { en territorio irregular }\end{array}$ \\
\hline & $\begin{array}{c}\text { Mejoras en } \\
\text { nutrición animal }\end{array}$ & & $\begin{array}{l}\text { Efecto del clima en la } \\
\text { producción de cultivos }\end{array}$ \\
\hline & Mejora de pasto & & $\begin{array}{c}\text { Contaminación sonora y } \\
\text { atmosférica }\end{array}$ \\
\hline & Caracterización molecular & \multirow{6}{*}{ Sector Salud } & Bioseguridad \\
\hline & $\begin{array}{l}\text { Aprovechamiento de } \\
\text { las tierras áridas }\end{array}$ & & Riesgos biológicos \\
\hline \multirow{4}{*}{$\begin{array}{c}\text { Tecnologías de } \\
\text { información } \\
\text { TIC's }\end{array}$} & $\begin{array}{l}\text { Estudio para determinar } \\
\text { la tarifa del taxímetro }\end{array}$ & & $\begin{array}{c}\text { Principales causas de } \\
\text { discapacidades prenatales }\end{array}$ \\
\hline & $\begin{array}{l}\text { Almacenamiento de datos } \\
\text { servicio en la nube }\end{array}$ & & Actualización medica \\
\hline & $\begin{array}{l}\text { Desarrollo de software de } \\
\text { aplicación en vigilancia }\end{array}$ & & $\begin{array}{l}\text { Prevalencia de la caries por } \\
\text { grupos de edad }\end{array}$ \\
\hline & $\begin{array}{c}\text { Warehouse, business intelligence, } \\
\text { arquitectura software }\end{array}$ & & Patología bucal y labio leporino \\
\hline
\end{tabular}


Relación investigación, innovación: el desarrollo de las empresas ecuatorianas con las universidades

\begin{tabular}{|c|c|c|c|}
\hline \multirow{8}{*}{$\begin{array}{l}\text { Tecnologías de } \\
\text { información } \\
\text { TIC's }\end{array}$} & Filemaker & \multirow{2}{*}{$\begin{array}{l}\text { Sector } \\
\text { Arquitectura }\end{array}$} & $\begin{array}{l}\text { Estudios de métodos } \\
\text { de construcción }\end{array}$ \\
\hline & $\begin{array}{l}\text { Nuevas Tecnologías enfocado } \\
\text { en seguridad informática }\end{array}$ & & Regeneración urbana \\
\hline & $\begin{array}{l}\text { Modelos educativos } \\
\text { a través de las Tics }\end{array}$ & \multirow{2}{*}{$\begin{array}{l}\text { Sector Artes y } \\
\text { Diseños }\end{array}$} & $\begin{array}{l}\text { Mejoramiento de } \\
\text { producción textil }\end{array}$ \\
\hline & $\begin{array}{c}\text { Automatización de } \\
\text { dispositivos electrónicos }\end{array}$ & & $\begin{array}{c}\text { Control de procesos industriales } \\
\text { y de productos para } \\
\text { controlar la calidad }\end{array}$ \\
\hline & $\begin{array}{c}\text { Automatización, sistematización } \\
\text { y líneas de producción }\end{array}$ & \multirow{3}{*}{$\begin{array}{c}\text { Sector } \\
\text { Empresarial }\end{array}$} & $\begin{array}{l}\text { Métodos tecnológicos } \\
\text { de control }\end{array}$ \\
\hline & $\begin{array}{c}\text { Proyectos de seguridad } \\
\text { electrónica }\end{array}$ & & $\begin{array}{l}\text { Desagregación tecnológica para } \\
\text { la sustitución de importaciones }\end{array}$ \\
\hline & $\begin{array}{l}\text { Fuentes alternativas de } \\
\text { producción de energía }\end{array}$ & & $\begin{array}{l}\text { Control de calidad } \\
\text { de polímeros }\end{array}$ \\
\hline & Reciclamiento de residuos & \multirow{3}{*}{$\begin{array}{c}\text { Sector } \\
\text { Comunicación }\end{array}$} & Print ready- Diseño \\
\hline \multirow{7}{*}{$\begin{array}{l}\text { Sector industrial } \\
\text { y petrolero }\end{array}$} & Gestión de procesos de BPM & & Marketing deportivo \\
\hline & $\begin{array}{l}\text { Perforación de pozos } \\
\text { sin contaminación }\end{array}$ & & Responsabilidad social \\
\hline & Técnicas de perforación & $\begin{array}{c}\text { Sector } \\
\text { Educación }\end{array}$ & $\begin{array}{l}\text { Apoyo seguimiento y } \\
\text { educación en adolecentes }\end{array}$ \\
\hline & Almacenamiento de hidrocarburos & & \\
\hline & software para almacenamiento & & \\
\hline & y transporte & & \\
\hline & $\begin{array}{c}\text { Automatización de la explotación } \\
\text { de recursos naturales }\end{array}$ & & \\
\hline
\end{tabular}

Fuente: Elaborado por los autores.

$\mathrm{Si}$ bien los requerimientos de investigación en cada uno de los sectores son muy diversos, en todos ellos es común la necesidad de investigación sobre innovación y actualización en procesos aplicados a sus ámbitos.

En la tabla 4 se señala cuáles son los mecanismos más apropiados para el proceso de transferencia del resultado de las investigaciones a sus beneficiarios; siendo los principales la asistencia técnica y servicios, la movilidad de personal y la alianza tecnológica (Ver tabla 4).

Tabla 4.

Mecanismos empleados por las IES para el proceso de transferencia de los resultados de investigación

\begin{tabular}{lcc}
\hline \multicolumn{1}{c}{ Mecanismo } & Empresas & Porcentaje \\
\hline Acuerdo de licencia & 16 & 4,2 \\
Asistencia técnica y servicios & 98 & 25,5 \\
Movilidad de personal & 52 & 13,5 \\
Cooperación tecnológica & 11 & 2,9 \\
Alianza tecnológica & 43 & 11,2 \\
No existe/No responde & 165 & 42,9 \\
No responde & 127 & 33,0 \\
Total & 385 & 100,0 \\
\hline
\end{tabular}

Fuente: Elaborado por los autores en base a la muestra de estudio en el 2015 


\section{Conclusiones}

El presente trabajo rescata como contribución fundamental a la ciencia, la relevancia de la investigación y la transferencia de conocimiento para el desarrollo empresarial y social, resaltando su campo práctico en la utilidad de la instituciones que programan la asignación de recursos para la ejecución e incentivo a la investigación, al igual que busca generar conciencia en la academia sobre su rol fundamental para el desarrollo de los grupos sociales de los cuales son parte, $\mathrm{y}$, de las empresas para generar un vínculo más fuerte con las universidades, capaz de promover su permanente innovación.

La investigación y la innovación tecnológica representan en el sector empresarial privado y público una fuente de crecimiento y desarrollo que estimula la producción, la generación de empleo y la creación de nuevos emprendimientos en favor del modelo económico de un país, aspecto que se ha reflejado en la investigación evidenciando que el desarrollo de la investigación en Ecuador ha promovido la innovación empresarial.

La interrelación entre los centros de educación superior y los sectores productivos y sociales, es cada vez más urgente, puesto que la academia es la encargada de generar los procesos de investigación que faciliten al empresario nuevas herramientas para mejorar e innovar en su práctica diaria, a su vez que provee de profesionales comprometidos con el desarrollo del país.

Se evidencia la necesidad de destinar recursos para impulsar la investigación e innovación empresarial con relación al aporte de las IES en proyectos conjuntos que impacten positivamente en la sociedad y le permitan al país competir. El mayor porcentaje de investigación lo realiza el Estado a través de los diferentes institutos del sector público, y en menor proporción los centros de educación superior, y dentro de estos los públicos. La mayor parte del financiamiento es vía presupuesto del estado a través de la SENESCYT y lo restante con recursos propios.

Las experiencias registradas sobre los procesos de vinculación entre IES y empresas, tanto del sector público como del privado en otros países y en el exterior son positivas, sin embargo, falta ampliar la cobertura a un número mayor de empresas. En el Ecuador las experiencias no son tantas, sin embargo en esta investigación se encontró que el $17 \%$ de las empresas han realizado proyectos de investigación en convenios con organizaciones del sector público o privadas sin fines de lucro. En el sector público recién se están firmando acuerdos de cooperación con otros países como es el caso de Australia, según consta en documentos de la SENESCYT (2013) y de la Red Ecuatoriana de Innovación, la cual formó un espacio para compartir experiencias sobre puntos de vista, conocer y analizar diversas temáticas e impulsar propuestas innovadoras, para que sean un aporte al desarrollo nacional. 
Finalmente, se reconocen como limitaciones de la investigación los recursos empleados para ampliar la muestra en el territorio ecuatoriano, el acceso a fuentes secundarias actualizadas y la disponibilidad de datos de las instituciones, con lo cual, se genera la posibilidad de futuras líneas de investigación que consideren la realización de estudios empíricos sobre la incidencia de la investigación en el desarrollo del país, y la relación de la producción académica con la mejora empresarial.

\section{Referencias}

Ayala, E. (2015). La investigación científica en las universidades ecuatorianas. Anales, Revista de la Universidad de Cuenca, 57, 61-72.

Berumen, S. (2011). Los Sistemas de Innovación en Europa. Madrid: Gráficas Dehon.

Cámara Zaragoza. (6 de Julio 2011). Qué diferencia existe entre los comceptos de investigación, desarrollo e innovación. Obtenido de: https:/www.camarazaragoza.com/faq/quediferencias-existen-entre-los-conceptos-de -investigacion-desarrollo-e-innovacion/

Cárdenas, S., Cabrero, E., y Arellano, D. (2014). La Dificil Vinculación Universidad Empresa en Mexico ¿Hacia la construcción de la Triple Hélice?. Mexico D.F: CIDE.

Carvajal, E. P. (2 de marzo de 2015). Investigación $y$ principales autores. Obtenido de: https://prezi.com/rda0plhjtl-i/investigacio n-y-principales-autores/

CEPAL. (2015). Estudio Económico de América Latina y el Caribe 2015: desafíos para impulsar el ciclo de inversión con miras a reactivar el crecimiento. Obtenido de: https://www.cepal.org/es/publicaciones/3 8713-estudio-economico-de-america-lati na-y-el-caribe-2015-desafios-para-impuls ar-el

CEPAL. (2010). Espacios Iberoamericanos, Vínculos entre Universidades y Empresas para el desarrollo Tecnológico. Obtenido de: https://www.cepal.org/es/publicaciones/141 7-espacios-iberoamericanos-vinculos-univer sidades-empresas-desarrollo-tecnologico

Colina, L. (2007). La investigación en la educación superior y su aplicabilidad social, Revista Educación, 13(25), 330-353.

Dallanegra, L. (mayo de 2004). La Universidad y la investigación científica en América Latina. Serie documentos de trabajo del IDICSO (23). Obtenido de:

http://csoc.usal.edu.ar/archivos/csoc/docs /idicso-sdti023.pdf

Garzón Castrillón, M. A., e Ibarra Mares, A. (junio de 2013). Innovación empresarial, difusión, definiciones y tipología: Una revisión de literatura. Revista Dimensión Empresarial, 11(1), 45-60.

González Molano, C., y Martínez Campos, J.L. (2014) Gerencia estratégica e innovación empresarial: referentes conceptuales. Revista Dimensión Empresarial, 12 (1), 107-116.

Morone, G. (s.f.). Métodos y técnicas de la investigación científica. Obtenido de: http://biblioteca.ucv.cl/site/servicios/document os/metodologias investigacion.pdf

Rojas, S. R. (2002). Investigación Social Teoría y Praxis. Mexico D.F: Plaza Valdés S.A.

SENESCYT. (12 de Agosto de 2013). Ecuador y Australia comparten experiencias de Ciencia, Tecnología e innovación. Recuperado el 04 de 03 de 2015, de: http://www.educacionsuperior.gob.ec/ecu ador-y-australia-comparten-experienciasde-ciencia-tecnologia-e-innovacion/ 
